\title{
Glasslike relaxation of labyrinthine domain patterns
}

\author{
Bert Reimann,* Reinhard Richter, and Ingo Rehberg \\ Physikalisches Institut, Universität Bayreuth, D-95440 Bayreuth, Germany
}

(Received 25 April 2001; published 27 February 2002)

\begin{abstract}
A spatial analysis of globally disordered (labyrinthine) stripe domain patterns in thin ferrimagnetic garnet films is applied to investigate the pattern evolution. After demagnetization of the sample we obtain a branched (fernlike) structure. By periodic modulation of the magnetic field the number of the branches diminishes and a labyrinthine pattern develops. We describe the evolution of the pattern by a measure extracted from the curvature of the border line of the magnetic domains. The relaxation of this measure is found to be nonexponential and can be described by the Kohlrausch-Williams-Watts law.
\end{abstract}

DOI: 10.1103/PhysRevE.65.031504

PACS number(s): 64.70.Pf, 75.50.Gg, 75.70.Kw

Labyrinths have been fascinating since the early days of Ariadne's thread. They are puzzling because of their short range order but long range disorder. Such structures can be found in a large number of nonequilibrium systems [1], and, in particular, the spiral defect chaos in Rayleigh-Bénard convection [2] provides a more recent example. Labyrinthine structures are characterized by the fact that lines do not end within the bulk of the sample, but rather at the boundary. Such patterns seem to be more prominent in equilibrium systems. Examples for magnetic systems are the labyrinthine patterns in magnetic liquids [3]. They can also be found on a microscopic scale, i.e., the ferromagnetic domains on a MnBi crystal [4]. Very similar magnetic structures can also be observed on a larger scale, namely, in ferrimagnetic garnet films [5].

Labyrinthine pattern in ferrimagnetic garnet films has been extensively analyzed using image processing techniques. In Ref. [6] they have been approximated by polygonal segments and successively statically analyzed. This procedure yields as observables the size and the orientation of polygonal plaquettes and the position of topological defects. With those tools it was shown that the transition from lamellar to labyrinthine patterns is reminscent of defect-mediated melting. These authors also suggested to regard labyrinths as a two-dimensional glass. In the present paper we extend this line of thought by demonstrating experimentally that the dynamics of the transition between disordered states in this system can indeed be described by the Kohlrausch-WilliamsWatt (KWW) law [7].

In particular, we investigate the evolution of disordered (fernlike) domain patterns shown in Fig. 1(a) to the labyrinthine state displayed in Fig. 1(c) in a ferrimagnetic garnet film. The order parameter used to quantify that transition is deduced from the curvature of the border line of the magnetic domains. We apply this measure to the evolution of the pattern triggered by a periodic modulation of the magnetic field. The relaxation of that order parameter is compared with a relaxation according to the Kohlrausch-Williams-Watt law.

For the experiment we use a commercial sample of ferrimagnetic garnet (FMG), which is situated in the center of a

*Email address: Bert.Reimann@uni-bayreuth.de electromagnetic coil. The experimental setup is shown in Fig. 2. The FMG sample is grown in a thin, single crystal (epitaxial) film, with the easy axis of magnetization perpendicular to the film. The dimensions of the film are 1.5 $\times 1.5 \mathrm{~mm}^{2}$ with a thickness of $8 \mu \mathrm{m}$. The material has the formula $\mathrm{Bi}_{0.6} \mathrm{Tm}_{2.4} \mathrm{Ga}_{1.15} \mathrm{Fe}_{3.85} \mathrm{O}_{12}$ and is transparent. The Faraday effect serves to visualize the magnetic domains: Polarized light illuminates the transparent FMG from below. Its polarization plane is turned by the local magnetization of the FMG. By means of the analyzer on top of the sample the magnetic domains are visible as bright or dark stripes.

To observe the pattern we use a microscope (Carl-ZeissJena) and a black and white charge-coupled-device (CCD)camera module (CCD Video Kameramodul/Leutron Vision $\mathrm{GmbH})$. The magnification of the microscope is selected to observe the whole pattern. The CCD-camera module is connected to the real-time frame grabber card (BFP-AT 90006.6 /Leutron Vision GmbH) mounted in the PC (Pentium PC 166 $\mathrm{MHz}$ ). We digitize the pattern on an array of $512 \times 512$ pixel with 256 gray scales.

In order to control the magnetic field we utilize an analogto-digital converter (WSB 100 Synthesizer Card/ QUANTECH Inc.). It is connected via an amplifier (eurotest/ ELBA-Modul-GmbH) and an ampere meter (5001 digital Multimeter/PREMA Präzisionselektronik $\mathrm{GmbH})$ to the coil around the sample.

As indicated in Fig. 1, our system shows two metastable states at zero field with a slightly different morphology. The disordered fernlike pattern shown in Fig. 1(a) is obtained by magnetizing the sample to saturation at $\left(\mathrm{B}_{s}=4.5 \mathrm{mT}\right)$ and a subsequent sudden demagnetization. The preparation of the labyrinthine pattern shown in Fig. 1(c) is obtained after magnetizing the sample with a slighly smaller field of $B$ $=4 \mathrm{mT}$. The magnetization is not completely saturated in this case, as indicated by the single white domain in Fig. 1(b). In the saturated situation, the whole image appears black, without any white domains. When lowering $B$ the single bright domain wrinkles up and invades the large dark domain. This process ends at zero magnetic field with the labyrinthine pattern of Fig. 1(c).

Such patterns result from a compromise between demagnetization and the energy of Bloch walls. The surprising feature that this might lead to complex patterns rather than straight stripes can be explained by an undulation instability 

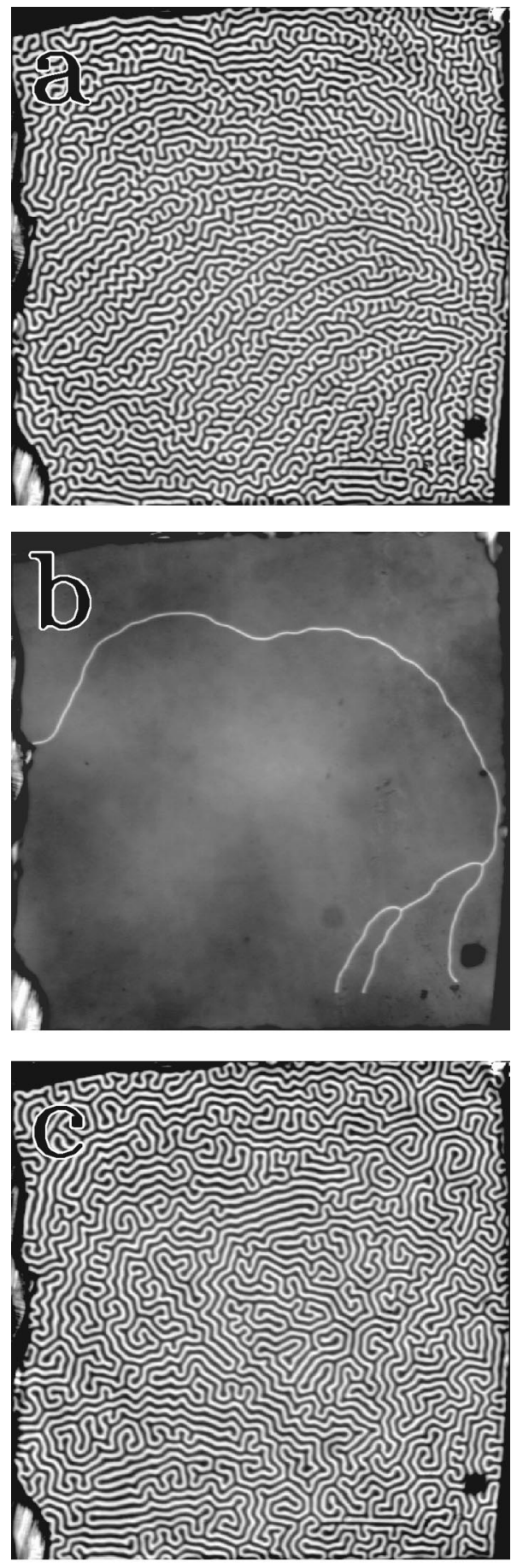

FIG. 1. Pattern evolution by slowly increasing the induction from $B=0 \mathrm{mT}$ (a) to $B=4 \mathrm{mT}$ (b) and decreasing it to $B=0 \mathrm{mT}$ (c).

of stripes, as demonstrated experimentally by Molho et al. [8] and subsequently explained theoretically by Sornette [9]. Patterns similar to the ones in Fig. 1 represent frustrated states and are metastable, i.e., every experimental run produces slightly different patterns, whose qualitative features seem similar.

For a quantitative characterization of the transition we extract the border line of the bright magnetic domains by first detecting the location of highest contrast within a grid of

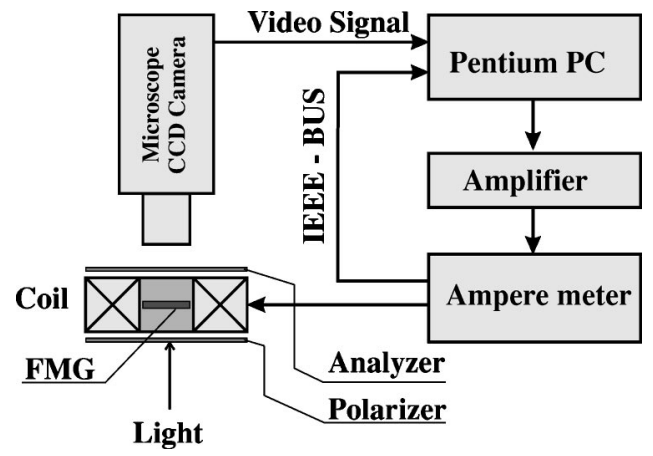

FIG. 2. Scheme of the experimental setup.

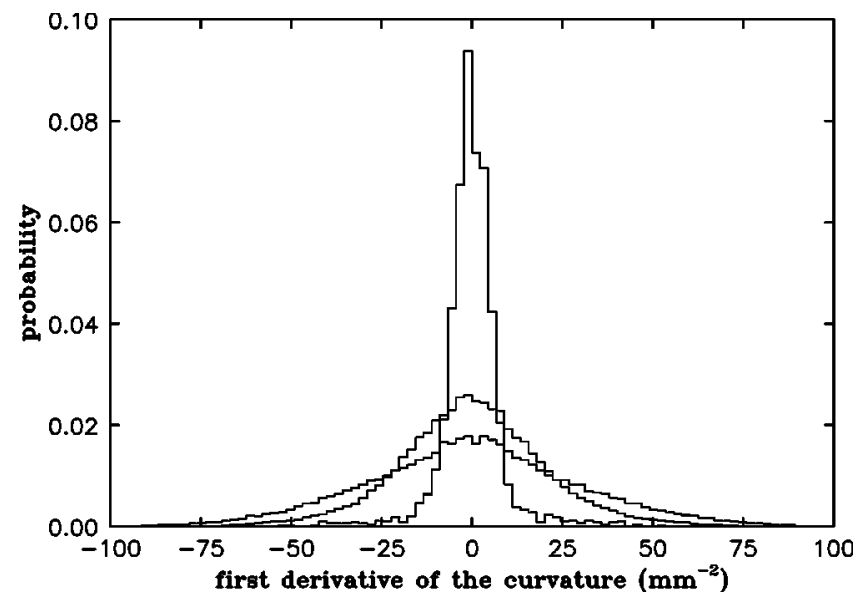

FIG. 3. Probability distribution of the first derivative of the borderline of the magnetic domains. The histograms (top to bottom) correspond to the pattern in Figs. 1(b), 1(c), and 1(a), respectively.

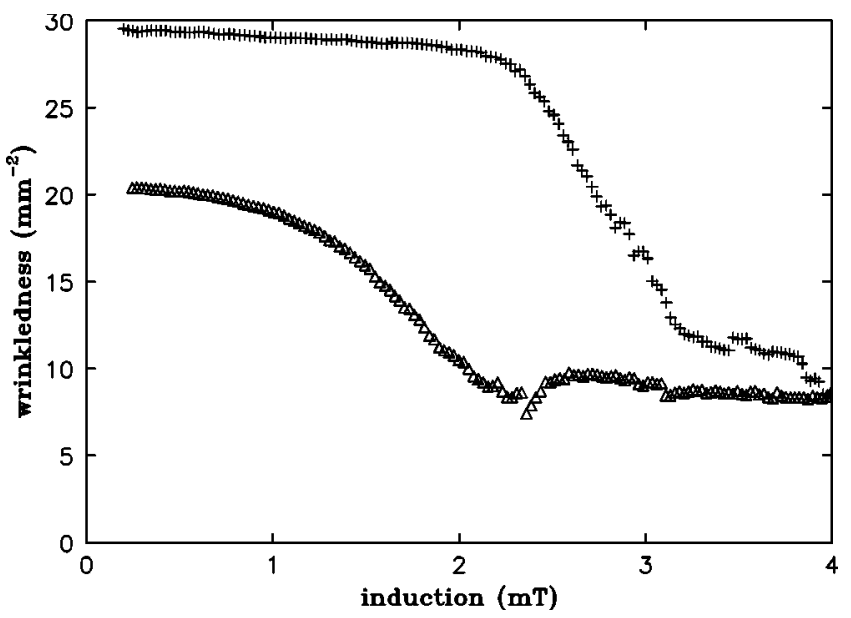

FIG. 4. The evolution of the wrinkledness for slowly increasing (crosses) and decreasing (triangles) the magnetic induction. 

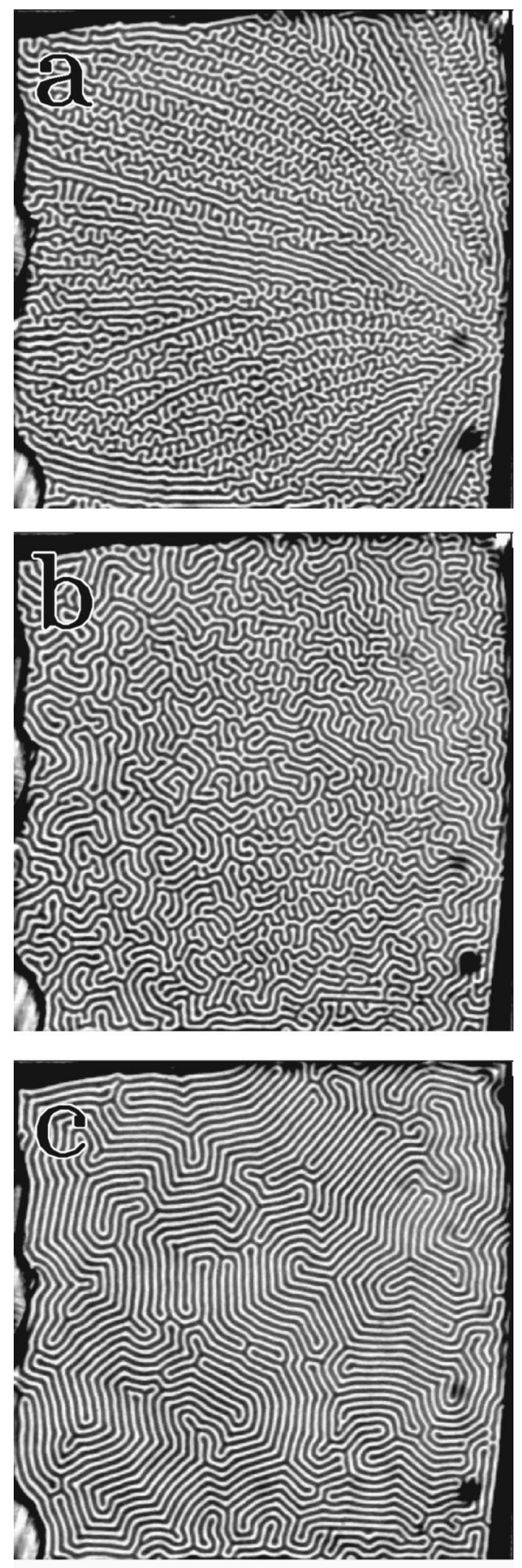

FIG. 5. Development of the domain pattern under periodic modulation according to Eq. (1) with $B_{0}=1.8 \mathrm{mT}$ and $f_{D}=1 \mathrm{~Hz}$. Elapsed time (a) $t=0 \mathrm{~s}$, (b) $t=50 \mathrm{~s}$, and (c) $t=5000 \mathrm{~s}$.

the image, which is refined by a factor of 2 compared to the original one by linear interpolation between the pixels. The curvature is subsequently derived from this discrete coordinates by using the discrete representation of the curvature. By this procedure the probability distribution of the first spatial derivative of the curvature of the border line is determined. Figure 3 displays the distributions estimated from the images shown in Fig. 1. Obviously the wrinkled structure of Fig. 1(a) is described by a broader distribution than the

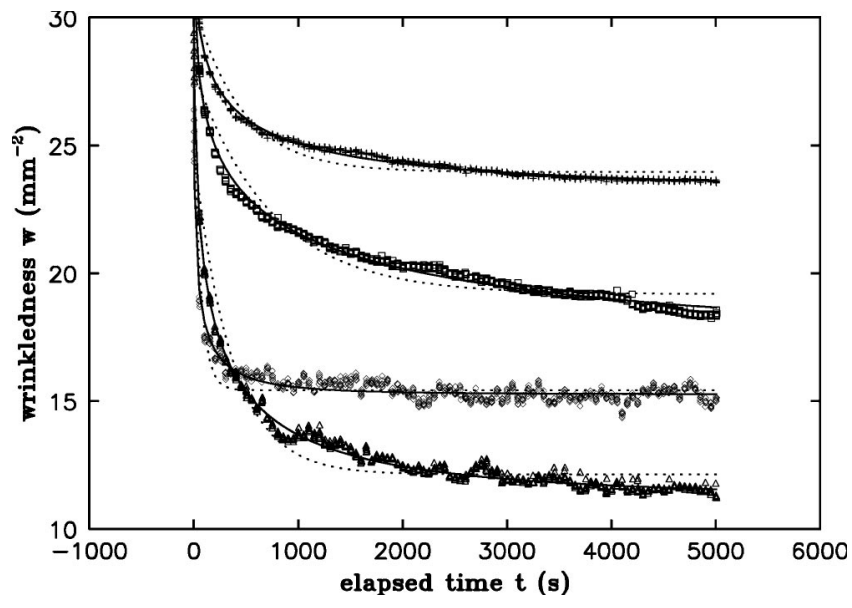

FIG. 6. Wrinkledness vs elapsed time. The crosses, squares, diamonds, and triangles represent different values of the amplitude: $B_{0}=0.8 \mathrm{mT}, 1.4 \mathrm{mT}, 2.0 \mathrm{mT}$, and $1.8 \mathrm{mT}$. The solid (dotted) lines represent fits to the KWW (exponential) relaxation.

smoother structure in Fig.1(c).

Because the standard deviation of the distribution is a measure for the degree of wrinkles of the pattern, we name this number "wrinkledness." It is plotted vs the induction $B$ in Fig. 4. Here the crosses (open triangles) denote the value for increasing (decreasing) the induction. In this way we are able to describe the transition from a fernlike structure to a labyrinthine one quantitatively.

The two morphologies under investigation are metastable states. In order to find if one state is energetically favored, we speed up the relaxation time between the two states into a range accessible to measurements by applying a timedependent magnetic induction

$$
B=\frac{1}{2} B_{0}\left(1-\cos \left(2 \pi f_{D} t\right)\right)
$$

where $B_{0}$ denotes the maximal induction and $f_{D}=1 \mathrm{~Hz}$ the driving frequency.

Under this periodic modulation the fernlike structure shown in Fig. 5(a) transforms via the morphology shown in Fig. 5(b) into the labyrinthine pattern of Fig. 5(c). During this transition the highly wrinkled structure is successively replaced by smooth patterns with lamellar short range order.

A quantitative description of this process is given in Fig. 6 . The relaxation of the wrinkledness is measured for four different values of $B_{0}$. For each value of $B_{0}$ the temporal evolution was recorded by taking 800 images over a time

TABLE I. Numerical values of the fit parameters as defined by Eq. (2).

\begin{tabular}{lcccc}
\hline \hline$B_{0}(\mathrm{mT})$ & $w_{0}\left(\mathrm{~mm}^{-2}\right)$ & $w_{\infty}\left(\mathrm{mm}^{-2}\right)$ & $\tau(\mathrm{s})$ & $\beta$ \\
\hline 0.4 & 35.28 & 23.19 & 183.5 & 0.36603 \\
0.8 & 35.70 & 16.50 & 391.0 & 0.30696 \\
1.8 & 32.10 & 11.20 & 153.0 & 0.40367 \\
2.0 & 29.13 & 15.27 & 32.0 & 0.39822 \\
\hline \hline
\end{tabular}


interval of about $2 \mathrm{~h}$. The solid lines give the fits to the Kohlrausch-Williams-Watt law

$$
w(t)=w_{0} e^{-(t / \tau)^{\beta}}+w_{\infty}\left(1-e^{-(t / \tau)^{\beta}}\right) .
$$

Here $w_{0}$ and $w_{\infty}$ denote the starting and end values of the wrinkledness $\mathrm{w}, \tau$ the characteristic relaxation time, and $\beta$ the fractional Kohlrausch exponent. The dotted lines stem from a fit with $\beta=1$ and represent the simple exponential law. Obviously, the stretched exponential KWW law characterizes the relaxation process better than the simple exponential law. As an alternative, we have also tried to fit the data by a power-law decay, which shows systematic deviations from the data, however.
The numerical values of the fit parameters are presented in Table I.

In conclusion, we have measured the evolution of a disordered state under temporal modulation. By using an order parameter derived from image analysis procedures we have shown that a relaxation law typical for microscopically disordered states can be observed for macroscopic patterns as well. Our results suggest that it seems adequate to associate the dynamics of magnetic domains with that of glasses.

It is a pleasure to thank J. Bläsing and G. Straßburger for stimulating discussions. The experiments were supportd by DFG through Grant No. Re 588/12.
[1] M.C Cross and P.C Hohenberg, Rev. Mod. Phys. 65, 851 (1993).

[2] E. Bodenschatz, W. Pesch, and G. Ahlers, Annu. Rev. Fluid Mech. 32, 709 (2000).

[3] R.E. Rosensweig, Ferrohydrodynamics (Cambridge University Press, Cambridge, 1985).

[4] S. Honda, S. Konishi, and T. Kusuda, Appl. Phys. Lett. 21, 421 (1972).
[5] M. Seul, L.R Monar, L. O'Gorman, and R. Wolfe, Science 245, 1557 (1991).

[6] M. Seul , L.R Monar, and L. O’Gorman, Philos. Mag. B 66, 471 (1992).

[7] S. Havlin and D. Ben-Avraham, Adv. Phys. 36, 695 (1987).

[8] P. Molho, J. Gouzerh, J.C.S. Levy, and J.L. Perteseil, J. Magn. Magn. Mater. 54-57, 857 (1986).

[9] D. Sornette, J. Phys. (France) 48, 151 (1989). 